\title{
Immune reactivity to Trypanosoma cruzi chimeric proteins for Chagas disease diagnosis in immigrants living in a non- endemic setting
}

Eva Dopico ${ }^{1}$, Rodrigo Pimenta Del-Rei², Bertha Espinoza ${ }^{3}$, Itziar Ubillos ${ }^{1}$, Nilson Ivo Tonin Zanchin ${ }^{4}$, Elena Sulleiro ${ }^{5}$, Zaira Moure ${ }^{5}$, Paola Alejandra Fiorani Celedon ${ }^{6}$, Wayner Vieira Souza ${ }^{7}$, Edimilson Domingos da Silva ${ }^{8}$,

Yara Miranda Gomes ${ }^{7}$ and Fred Luciano Neves Santos ${ }^{9^{*}}$

\begin{abstract}
Background: Chronic Chagas Disease (CD) diagnosis is based on serological methods employing crude, semipurified or recombinant antigens, which may result in low sensitivity or cross-reactivity. To reduce these restrictions, we developed a strategy involving use of molecules containing repetitive fragments of Trypanosoma cruzi conserved proteins. Diagnostic performance of IBMP-8.1 and IBMP-8.4 chimeric antigens (Molecular Biology Institute of Paraná - IBMP in Portuguese acronym) was assessed to diagnose T. cruzi-infected and non-infected immigrants living in Barcelona (Spain), a non-endemic setting for Chagas disease.

Methods: Reactivity of IBMP-8.1 and IBMP-8.4 was assessed using an in-house automated ELISA with 347 positive and 331 negative individuals to Chagas disease. Antigenic cross-reactivity was measured with sera samples from pregnant women with Toxoplasma gondii $(n=98)$ and Zika virus $(n=75)$ antibodies.

Results: The area under the curve values was 1 and 0.99 for the IBMP-8.1 and IBMP-8.4 proteins, respectively, demonstrating excellent diagnostic accuracy. The reactivity index was higher for IBMP-8.1 than IBMP-8.4 in positive samples and no significant difference in reactivity index was observed in negative samples. Sensitivity ranged from 99.4\% for IBMP-8.1 to $99.1 \%$ for IBMP-8.4 and was not statistically different. Specificity for IBMP-8.1 reached 100 and $99.7 \%$ for IBMP-8.4, both nearly 100\% accurate. No antigenic cross-reactivity was observed and reactivity index was similar to that for negative Chagas disease individuals.
\end{abstract}

Conclusions: Our results showed an outstanding performance of IBMP-8.1 and IBMP-8.4 chimeric antigens by ELISA and suggest both chimeric antigens could also be used for Chagas disease diagnosis in immigrants living in nonendemic settings.

Keywords: Chagas disease, Trypanosoma cruzi, Chimeric antigens, Immunoassay, Accuracy

\footnotetext{
* Correspondence: fred.santos@bahia.fiocruz.br

Preliminary results were presented at XXIV Latin American Congress of

Parasitology (FLAP XXIV); 10 December 2017; School of Medicine; Santiago,

Chile.

${ }^{9}$ Gonçalo Moniz Institute, Oswaldo Cruz Foundation, Salvador, Bahia, Brazil

Full list of author information is available at the end of the article
}

(c) The Author(s). 2019 Open Access This article is distributed under the terms of the Creative Commons Attribution 4.0 International License (http://creativecommons.org/licenses/by/4.0/), which permits unrestricted use, distribution, and reproduction in any medium, provided you give appropriate credit to the original author(s) and the source, provide a link to the Creative Commons license, and indicate if changes were made. The Creative Commons Public Domain Dedication waiver (http://creativecommons.org/publicdomain/zero/1.0/) applies to the data made available in this article, unless otherwise stated. 


\section{Background}

Chagas disease (CD) is a life-threatening infection caused by hemoflagellate protozoa Trypanosoma cruzi, generating an estimated of 14,000 deaths every year [1] and morbidity in 5.7 to 9.4 million people in the continental Western Hemisphere [2, 3]. The epidemiological pattern of CD has undergone substantial changes in last decades as a consequence of control campaigns in endemic countries, which have reduced vectorial and transfusional transmission [4]. Increasing international migration flows and more affordable traveling conditions from Latin America to non-endemic areas have contributed to epidemiology changes $[5,6]$. CD is no longer limited exclusively to the impoverished rural regions of Latin America; it is transformed into a global health concern affecting people worldwide in both endemic and non-endemic countries and placing 100 million people at risk for acquiring the infection [7].

In Spain, there are more than 6 million immigrants, and more than 2 million (38\%) are coming from CD endemic Latin America countries [8], posing CD as a public health challenge [9]. Indeed, Ecuador, Bolivia, and Argentina are the predominant areas of origin [7]. The diversity of the geographic areas leads to another challenge: the need for an accurate diagnostic test capable of identifying individuals infected with different $T$. cruzi strains. The high genetic variability of $T$. cruzi can be responsible for false negative results [10]. These false negative results could be avoided by using synthetic chimeric antigens with repetitive fragments of antigenic T. cruzi proteins for the detection of specific antibodies [11-14].

We performed ELISA [15, 16] and liquid microarray [17] to assess the potential diagnostic of four chimeric proteins, IBMP-8.1, IBMP-8.2, IBMP-8.3, and IBMP-8.4, to identify $T$. cruzi-infected individuals from several Brazilian endemic (Bahia, Goiás, Minas Gerais, and Pernambuco states; Brazil) and non-endemic settings (Paraná state; Brazil). These chimeric antigens were composed of immunodominant and conserved sequences, as described previously [15]. We obtained high-performance values and low cross-reactivity to Leishmania spp., a pathogen showing relatively high antigenic similarity to T. cruzi $[15,17]$. Imprecision analyses showed that IBMP chimeric antigens are highly reproducible and IBMP-8.1 and IBMP-8.4 presented the highest performance values among the evaluated antigens. In this study, we endeavored to conduct an evaluation of the diagnostic performance of IBMP-8.1 and IBMP-8.4 chimeric antigens employing ELISA to diagnose $T$. cruzi-infected and non-infected immigrants living in Barcelona (Spain), a non-endemic setting for the CD.

\section{Methods}

We used the methodology previously described by Santos et al. [18] and Brito et al. [19].

\section{Study samples}

We employed anonymized human sera from individuals diagnosed at the Laboratory Clínic l'Hospitalet-Laboratori Clínic Territorial Metropolitana Sur, Catalan Institute of Health (Barcelona-Spain). The minimum sample with a 95\% confidence interval, an absolute expected error of $1.1 \%$ and sensitivity of $99 \%$ was 315 sera from non-infected and 315 from T. cruzi-infected individuals. We included sera from 331 non-infected and 347 T. cru$z i$-infected Latin American individuals living in Barcelona (Fig. 1). The sample selection was based on non-reactivity and reactivity by two serological assays: $\mathrm{ORTHO}^{\circ} T$. cruzi ELISA Test System (Ortho Clinical Diagnostics Inc., Raritan, USA), which employs T. cruzi whole cell lysate antigen; and Bioelisa CHAGAS (Biokit S.A., Barcelona, Spain) or BIO-FLASH ${ }^{\bullet}$ Chagas (automated chemiluminescent assay; Biokit S.A., Barcelona, Spain), the two latter composed by recombinant $T$. cruzi antigens. Samples with repeatedly discrepant results between both tests or inconclusive in one of them (or in gray zone) were defined as serodiscordant. Each sample was given an identifier code in the laboratory to ensure a blinded analysis. Antigenic cross-reactivity was assessed with sera samples from Latin American pregnant women with Toxoplasma gondii $(n=98)$ and Zika virus (ZIKV) antibodies $(n=75)$. Pregnant women sera samples were employed in this study due to the availability of the biological material in Laboratory Clínic l'Hospitalet-Laboratori Clínic Territorial Metropolitana Sur serum bank. Study participants were mostly from Bolivia (97.3\%), but there were individuals from other CD endemic countries (Fig. 1).

\section{Recombinant antigens}

Proteins were expressed in Escherichia coli $\mathrm{Bl} 21$ star DE3 and purified from the soluble fraction of the total extract of bacterial lysate. Both IBMP-8.1 and IBMP-8.4 antigens were purified by IMAC resin first, and the best fractions dialyzed for buffer exchange and salt reduction before following a second liquid chromatography step. The second purification was conducted on ionic exchange and heparin columns, respectively. Plasmidial construct has already been described in Santos et al. [18].

\section{Immunoassays}

Assays were conducted according to previous reports $[14,16]$. Briefly, polystyrene "Maxisorp" 96-well microplates (Nunc, Roskilde, Denmark) were coated with 25.0 ng of IBMP-8.1 and IBMP-8.4 per well diluted in coating buffer $(0.05 \mathrm{M}$ carbonate-bicarbonate, $\mathrm{pH}$ 9.6). Microplates were blocked with Well Champion reagent (Kem-En-Tec, Taastrup, Denmark) according to the manufacturer's instructions. Serum samples were diluted in $0.05 \mathrm{M}$ phosphate-buffered saline ( $\mathrm{pH} 7.2)-0.5 \%$ Tween 20 


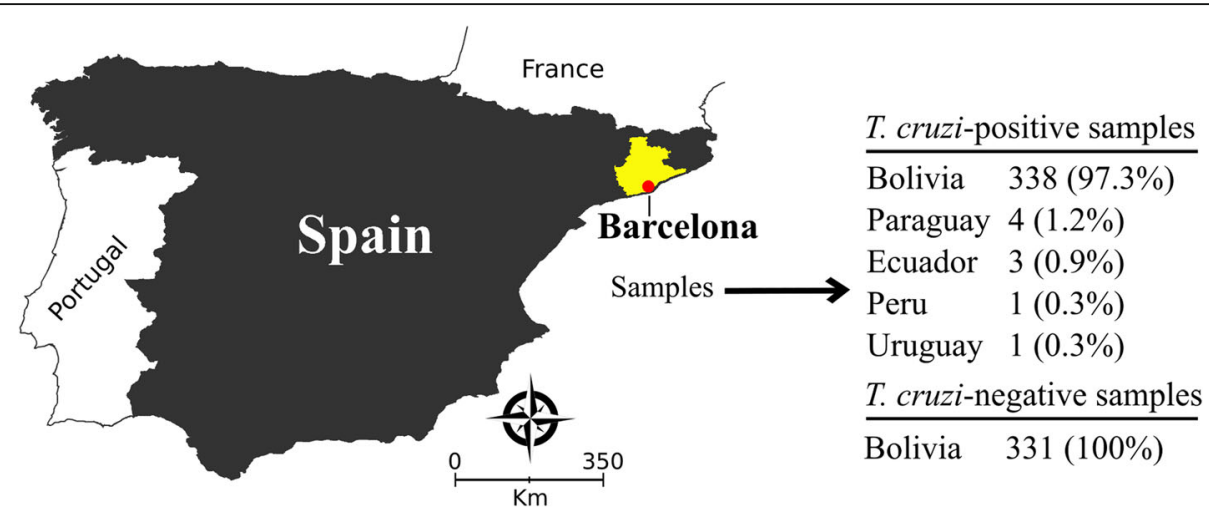

Fig. 1 T. cruzi-positive and negative samples selected from Latin American immigrants living in Barcelona-Spain

(PBS-T), and $100 \mu \mathrm{l}$ was added to each well. After $60 \mathrm{~min}$ of incubation at $37^{\circ} \mathrm{C}$, microplates were washed in PBS-T to remove unbound antibodies. HRP conjugated goat anti-human IgG (Bio-Manguinhos, FIOCRUZ, Rio de Janeiro, Brazil) was diluted 1:40,000 in PBS-T, and $100 \mu \mathrm{l}$ were then added to each well, and the microplates were incubated for $30 \mathrm{~min}$ at $37^{\circ} \mathrm{C}$. Wells were washed five times and the immune complexes were revealed by the addition of $100 \mu \mathrm{l} \mathrm{TBM}$ substrate (tetramethyl-benzidine; Kem-En-Tec, Taastrup, Denmark). After a new cycle of incubation (10 min at RT in the dark), the reaction was stopped by adding $50 \mu \mathrm{l} 5 \mathrm{~N} \mathrm{H}_{2} \mathrm{SO}_{4}$, and the absorbance was measured at $450 \mathrm{~nm}$. The protocols were automatized and the runs carried out in an automated microplate immunoanalyser (BEST $2000^{\circ}$, Biokit, Werfen Group Barcelona, Spain). The blank readings (buffer dilution) was subtracted from all other values.

\section{Statistical analysis}

Data were coded and entered using computer graphic software (GraphPad Software Inc., La Jolla, CA, USA). Descriptive data were presented in the form of geometric means \pm standard deviation. Shapiro-Wilk test, followed by Student's t-test, was used to test data normality. When assumed homogeneity was not confirmed, Wilcoxon's signed rank test was adopted. Cut-off values were determined under the receiver operating characteristic curve (ROC) analyzing the whole serum panel. For data normalization, all results were expressed by plotting values in an indexed format, calculated as the ratio between a given sample's optical density (OD) and the cut-off OD values respective to each assay. Under this index, referred to as a reactivity index (RI), all results $\geq 1.00$ were considered positive. When a sample's RI value was $1.0 \pm 10 \%$, the result was considered as indeterminate (i.e., in the grey zone), and these samples were deemed inconclusive. The test performances were computed using a dichotomous approach and compared regarding sensitivity, specificity, and accuracy [20]. Confidence interval was set to $95 \%$ and $p<0.05$ was considered as statistically significant. A study workflow (Fig. 2) is provided according to the STARD guidelines [21]. Digital map (Fig. 1) was acquired from the Brazilian Institute of Geography and Statistics (IBGE) cartographic database in shapefile (.shp), which were formatted and analyzed using TerraView version 4.2, public-access software provided by the National Institute for Space Research from Brazil (www.dpi.inpe.br/ terraview).

\section{Results}

\section{Diagnostic performance}

The reactivity index and assay performance parameters found for the IBMP-8.1 and IBMP-8.4 chimeric antigens are illustrated in Fig. 3. Based on 678 samples from non-infected and T. cruzi-infected individuals, the AUC (area under the curve) values were 1.000 and 0.9998 for IBMP-8.1 and IBMP-8.4 proteins, respectively, demonstrating excellent overall diagnostic accuracy. The IBMP-8.1 chimera yielded the highest diagnostic accuracy among T. cruzi-infected individuals, and no significant difference was observed between chimeric antigens in non-infected individuals. Sensitivity was $99.4 \%$ for IBMP-8.1 and 99.1\% for IBMP-8.4. Only one positive sample was simultaneously false negative when assayed by IBMP-8.1 and IBMP-8.4 chimeric antigens. Specificity of IBMP-8.1 achieved 100 and $99.7 \%$ for IBMP-8.4. Both proteins showed an accuracy of nearly $100 \%$. No statistically significant differences in sensitivity or specificity scores were found between the chimeric antigens. Using RI values of $1.0 \pm 0.10$ as the grey zone inconclusive interval, we observed two positive and one negative sample fell in the inconclusive space using IBMP-8.1 while two positive samples to IBMP-8.4 presented similar behavior. Overall, the number of inconclusive results was 0.44\% for IBMP-8.1 and 0.29\% for IBMP-8.4 (Fig. 3). No sample fell concomitantly inside the grey zone for IBMP-8.1 and IBMP8.4 chimeric antigens. 


\section{Eligible Samples}
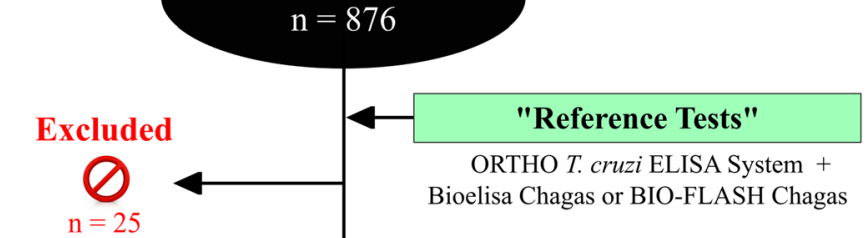

(Reason: Inconclusive or discordant results)

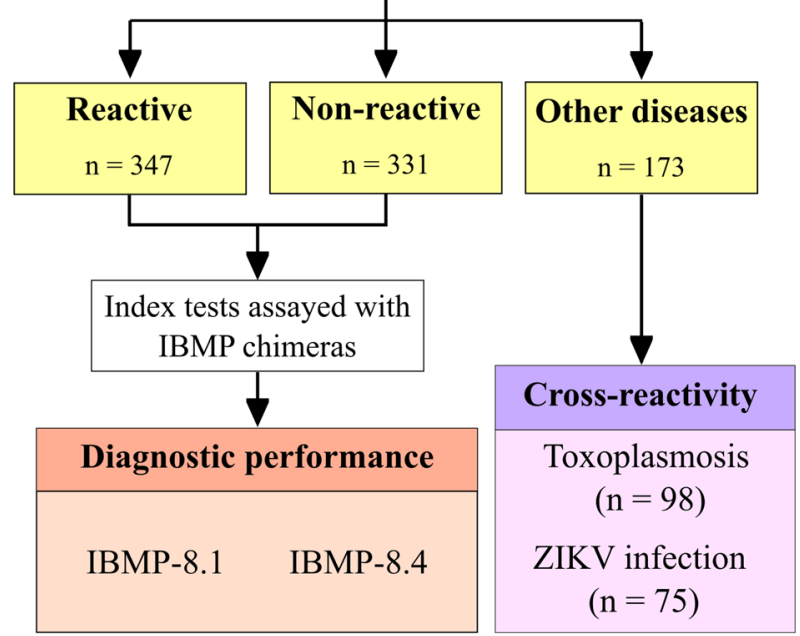

Fig. 2 Study workflow for testing IBMP T. cruzi chimeras. RI, reactivity index; ZIKV, Zika virus

IBMP-8.1

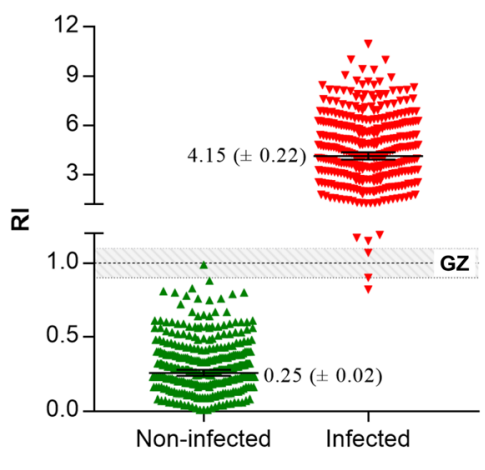

AUC: $1.0000( \pm 0.0001)$

Sen (\%): $99.4(97.9-99.8)$

Spe (\%): 100 (98.9-100)
Acc (\%): 99.7 (98.9-99.9)

IBMP-8.4

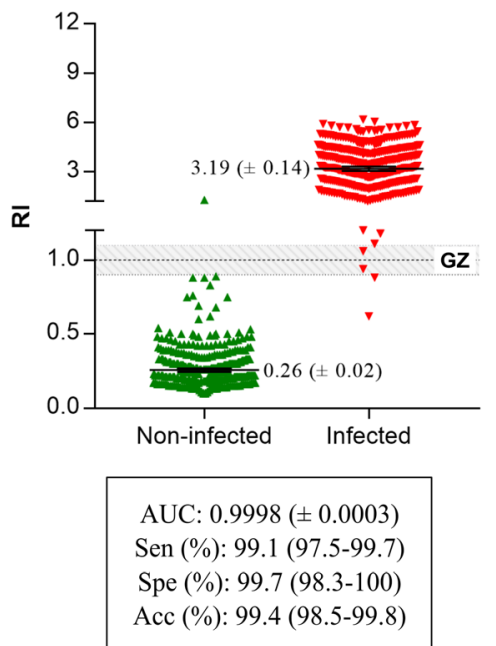

Fig. 3 Reactivity index (RI) was obtained with serum from non-infected and T. cruzi-infected immigrants living in Barcelona-Spain. The cut-off value is $\mathrm{RI}=1.0$ and the grey zone is $\mathrm{RI}=1.0 \pm 0.10$. Lines and whiskers represent geometric means ( $\pm 95 \% \mathrm{Cl}$ ). AUC, area under curve; $\mathrm{GZ}, \mathrm{grey}$ zone; Sen, sensitivity; Spe, specificity; Acc; accuracy 


\section{Antigenic cross-reactivity}

The RI values for sampled with $T$. gondii and ZIKV antibodies were identical to that found for non-infected individuals, indicating extremely low reactivity. No false-positive or inconclusive samples were found with IBMP chimeric antigens (Fig. 4).

\section{Discussion}

Diagnosis of chronic CD is not a simple task due to the high genetic diversity of $T$. cruzi, which might lead to misdiagnosis [22]. In fact, T. cruzi parasite has remarkable genetic heterogeneity, it is classified into seven evolutionary genetic groups or discrete typing units (DTUs) termed TcI-TcVI and TcBat, with sub-classifications for regional strains [22-24]. Regional differences in sensitivity of serological tests had been reported, leading to negative $C D$ diagnosis, mostly in non-endemic countries that receive immigrants from several endemic areas [25-28]. Therefore, a serological test should be able to diagnose CD regardless of the T. cruzi antigenic heterogeneity. The main benefit in the use of chimeric antigens as antigenic matrix is the increased repertoire of epitopes in comparison to non-chimeric recombinant antigens, reducing the number of false negative results. In previous studies, our group analyzed the IBMP performance to diagnose $T$. cruzi-infected individuals in several endemic and non-endemic geographical areas from Brazil $[14,15]$, a country where TcII is predominant [10]. Here, we used two chimeric antigens to capture specific anti-T. cruzi antibodies in the sera of Latin American immigrants living in Barcelona/ Spain, a non-endemic setting for $\mathrm{CD}$. The majority of CD-positive samples were collected from Bolivian immigrants, where $\mathrm{TcV}$ is the most common DTU found in Bolivia [10] and predominates in Bolivian immigrants living in Barcelona [29]. Based on the result from previous studies, we suggest that IBMP-8.1 and IBMP-8.4 antigens are able to diagnose chronic Chagas disease in areas with predominance of $\mathrm{TcII}$ and $\mathrm{TcV}$ genetic groups.

The assays exhibited high diagnostic accuracy values as demonstrated by AUC (nearly 100\%), indicating a substantial discriminative power between negative and chronic CD-positive samples. Similar results were previously found in samples from several Brazilian settings both by ELISA (AUC > 99.7\%) [14] and liquid microarray (AUC > 99.1\%) [17]. Moreover, the reactivity index from IBMP-8.1 and IBMP-8.4 chimeric antigens, achieved from immigrants living in a non-endemic setting with $\mathrm{CD}$, was higher than those previously obtained for Brazilian samples [14]. It is interesting to note that no differences were observed concerning negative samples. Further studies need to evaluate the performance of the chimeric antigens in settings where other DTUs are predominant, i.e., Argentina, Mexico and Costa Rica.

The present study showed high sensitivity and specificity for both IBMP-8.1 and IBMP-8.4, similar to those found previously using Brazilian samples $[14,15]$. Also, the chimeric antigens were found to be nearly $100 \%$ accurate, suggesting that the number of misdiagnoses was negligible. In fact, only two and three CD-positive samples were misclassified when assayed with IBMP-8.1 and IBMP-8.4, respectively. In previous studies we evaluated the increase of sensitivity values and the RI signal using a multiplex methodology [17] or the equimolar mixture of the antigens [15], however no gains of were achieved. Hence, we believe that the lack of reactivity from these samples could be due to host immunological reasons or low levels of antibodies on the sera. In CD-negative samples, only one sample was classified as false-positive by IBMP-8.4 antigen. Although this sample presented low signal to IBMP-8.4 (1.28), it was classified as negative when assayed by commercial tests $(\mathrm{RI}<0.25)$ and by the IBMP-8.1 chimera (0.22).

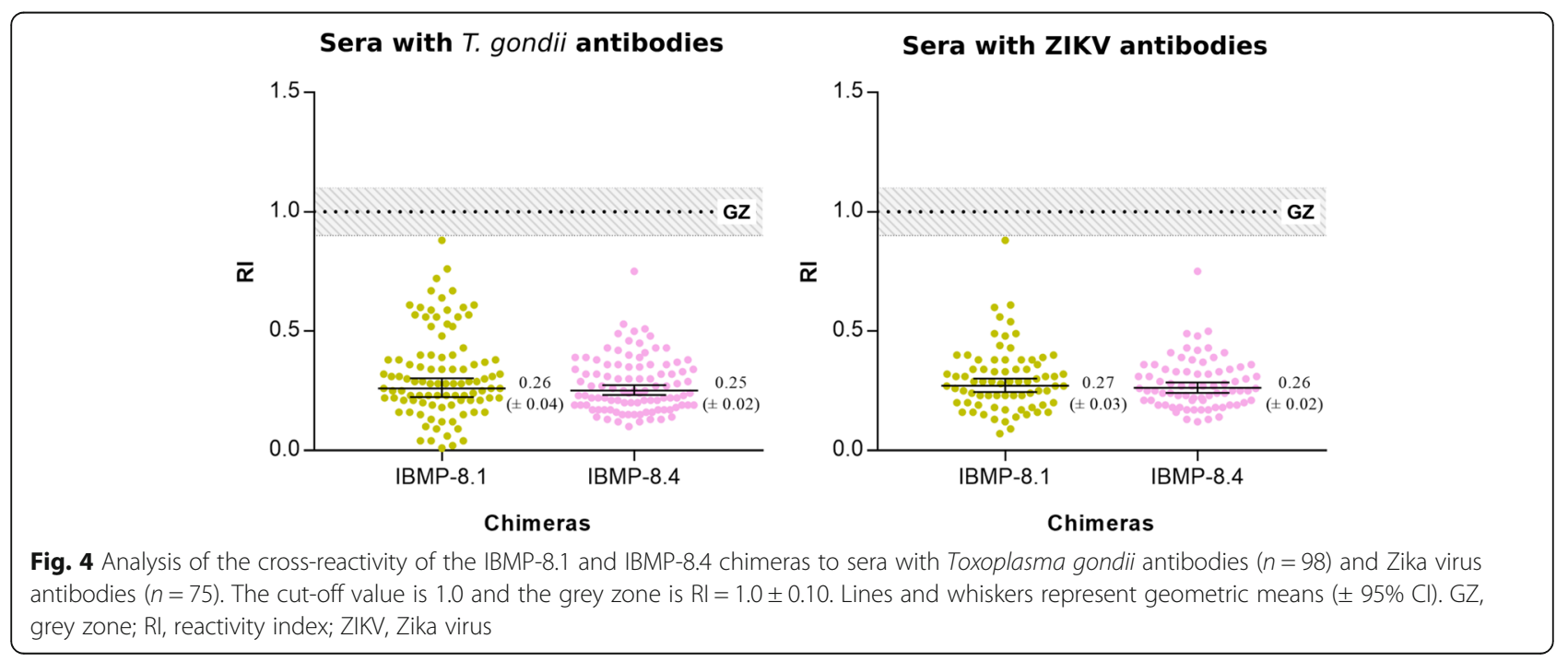


No cross-reactivity of IBMP chimeric antigens against antibodies of $T$. gondii and ZIKV was observed. Indeed, previous studies have shown extremely low reactivity of IBMP chimeric antigens for several infectious diseases, even for Leishmania spp. [15, 17], a pathogen phylogenetically similar to T. cruzi. T. gondii and ZIKV positive samples were used in this study due to serum bank availability and because these infectious diseases did not assay before using IBMP proteins.

\section{Conclusion}

Our results showed a remarkable performance of IBMP-8.1 and IBMP-8.4 chimeric antigens by ELISA and suggest both antigens could also be used for $C D$ diagnosis in immigrants living in non-endemic settings. The high accuracy of IBMP-8.1 and IBMP-8.4 chimeric antigens suggests that they are useful for $\mathrm{CD}$ diagnosis in individuals infected with other DTUs.

\section{Abbreviations \\ Acc: Accuracy; AUC: Area under the curve; CD: Chagas disease; DTU: Discrete typing unit; GZ: Grey zone; HRP: Horseradish peroxidase; IBMP: Molecular Biology Institute of Paraná (IBMP in Portuguese acronym); OD: Optical density; PBS: Phosphate-buffered saline; PBS-T: Phosphate-buffered saline- 0.5\% Tween 20; Rl: Reactivity index; ROC: Receiving operator curve; Sen: Sensitivity; Spe: Specificity; STARD: Standards for reporting of diagnostic accuracy studies; T. cruzi: Trypanosoma cruzi; T. gondii: Toxoplasma gondii; TBM: Tetramethyl-benzidine; ZIKV: Zika virus}

\section{Acknowledgements}

The authors would like to address a special thanks to Ignacio Martínez (Departmento de Immunologia, Instituto de Investigaciones Biomedicas, Universidad Nacional Autonoma de Mexico) and Jesus Almeda (Unitat de Suport a la Recerca, Direcció d'Atenció Primària Costa de Ponent-IDIAP J Gol) for their critical review. We also thank the staff at the Laboratori Clínic Hospitalet (Laboratori Clínic Territorial Metropolitana Sud, Catalan Institute of Health, Barcelona/Spain), especially Elisabeth Castro, as well as Montse Graells and Cristina Fernández for help with sample collection and laboratory analysis.

\section{Availability of the data and materials}

The datasets used and analyzed during the current study are available from the corresponding author on reasonable request.

\section{Funding}

This research project was funded by a Gonçalo Moniz Institute, Oswaldo Cruz Foundation (Fiocruz/BA). The funder was not involved in the design of the study, in the collection, analysis and interpretation of the data, or in writing the manuscript.

\section{Authors' contributions}

FLNS and ED were involved in the design and operational management of the study. FLNS and WVS were the lead study statistician. ED and RPDR were principal investigators in this study. All authors (ED, RPDR, BE, IU, NITZ, ES, ZM, PAFC, WVS, EDS, YMG, and FLNS) contributed to the interpretation of the data, contributed to this publication and approved the final manuscript for submission. All authors (ED, RPDR, BE, IU, NITZ, ES, ZM, PAFC, WVS, EDS, YMG, and FLNS) had access to the study data and are responsible for the veracity and completeness of the data reported.

\section{Author information}

FLNS is investigator in public health at Gonçalo Moniz Institute, Oswaldo Cruz Foundation (Fiocruz), Brazil.

\section{Ethics approval and consent to participate}

Ethical permission was sought and granted by Clinical Research Ethics Committee of Bellvitge University Hospital (Barcelona, Spain; IRB OHRP:
IRB00005523), and was carried out in accordance with the guidelines of the Helsinki Declaration. With the purpose of protecting the patient's private information, the Clinical Research Ethics Committee of Bellvitge University Hospital approved that the samples were anonymized so that the investigators do not have access to patient's private information, therefore, avoiding the requirement of verbal or written consent. This manuscript has also been revised for its publication by the Clinical Research Ethics Committee of Bellvitge University Hospital.

\section{Consent for publication}

Not applicable.

\section{Competing interests}

The authors declare that they have no competing interests.

\section{Publisher's Note}

Springer Nature remains neutral with regard to jurisdictional claims in published maps and institutional affiliations.

\section{Author details}

${ }^{1}$ Laboratori Clínic Territorial Metropolitana Sud, Catalan Institute of Health, Barcelona, Catalonia, Spain. ${ }^{2}$ Faculty of Technology and Sciences of Bahia, Salvador, Bahia, Brazil. Innstituto de Investigaciones Biomédicas, Departamento de Inmunología, Universidad Nacional Autónoma de México, Ciudad de México, Mexico. ${ }^{4}$ Carlos Chagas Institute, Oswaldo Cruz Foundation, Curitiba, Paraná, Brazil. ${ }^{5}$ Microbiology Department, Vall d'Hebron University Hospital, PROSICS, Barcelona, Catalonia, Spain. ${ }^{6}$ Molecular Biology Institute of Paraná, Curitiba, Paraná, Brazil. ${ }^{7}$ Aggeu Magalhães Institute, Oswaldo Cruz Foundation, Recife, Pernambuco, Brazil. ${ }^{8}$ Immunobiological Technology Institute, Oswaldo Cruz Foundation, Rio de Janeiro, Brazil. ${ }^{9}$ Gonçalo Moniz Institute, Oswaldo Cruz Foundation, Salvador, Bahia, Brazil.

Received: 24 September 2018 Accepted: 4 March 2019 Published online: 12 March 2019

\section{References}

1. Hotez PJ, Dumonteil E, Woc-Colburn L, Serpa JA, Bezek S, Edwards MS, et al. Chagas disease: "the new HIV/AIDS of the Americas". PLoS Negl Trop Dis. 2012;6(5):e1498.

2. World Health Organization. Chagas disease in Latin America: an epidemiological update based on 2010 estimates. Wkly Epidemiol Rec. 2015;90(6):33-43.

3. Global Burden of Disease Study 2013 Collaborators, Collaborators GB of DS 2013. Global, regional, and national incidence, prevalence, and years lived with disability for 301 acute and chronic diseases and injuries in 188 countries, 1990-2013: a systematic analysis for the global burden of disease study 2013. Lancet. 2015;386(9995):743-800.

4. Soriano Arandes A, Muñoz Gutierrez J, Vergés Navarro M, Castells Doménech C, Portús Vinyeta M, Gascón Brustenga J. Prevalence of Chagas disease in the Latin American immigrant population in a primary health Centre in Barcelona (Spain). Acta Trop. 2009;112(2):228-30.

5. Angheben A, Boix L, Buonfrate D, Gobbi F, Bisoffi Z, Pupella S, et al. Chagas disease and transfusion medicine: a perspective from non-endemic countries. Blood Transfus. 2015;13(4\$):540-50.

6. Pane S, Giancola ML, Piselli $P$, Corpolongo A, Repetto E, Bellagamba R, et al. Serological evaluation for Chagas disease in migrants from Latin American countries resident in Rome, Italy. BMC Infect Dis. 2018;18(1):212.

7. Strasen J, Williams T, Ertl G, Zoller T, Stich A, Ritter O. Epidemiology of Chagas disease in Europe: many calculations, little knowledge. Clin Res Cardiol. 2014;103(1):1-10

8. Eurostat. Total number of long-term immigrants arriving into the reporting country during the reference year. Available from: http://ec.europa.eu/ eurostat/tgm/table.do?tab=table\&init=1\&plugin $=1$ \&pcode $=$ tps00176\&language $=$ fr. Accessed 16 Oct 2017

9. Instituto Nacional de Estadística. Población residente por fecha, sexo, edad nacionalidad (agrupación de países) y lugar de nacimiento (agrupación de países). Available from: http://www.ine.es/jaxiPx/tabla.do?type=pcaxis\&path=/ t20/p321/serie/2001/l0/\&file=01005.px\&L=1. Accessed 18 Oct 2017.

10. Zingales B. Trypanosoma cruzi genetic diversity: something new for something known about Chagas disease manifestations, serodiagnosis and drug sensitivity. Acta Trop. 2018;184:38-52. 
11. Camussone C, Gonzalez V, Belluzo MS, Pujato N, Ribone ME, Lagier CM, et al. Comparison of recombinant Trypanosoma cruzi peptide mixtures versus multiepitope chimeric proteins as sensitizing antigens for immunodiagnosis. Clin Vaccine Immunol. 2009:16(6):899-905.

12. Hernández P, Heimann M, Riera C, Solano M, Santalla J, Luquetti AO, et al. Highly effective serodiagnosis for Chagas' disease. Clin Vaccine Immunol. 2010;17(10):1598-604.

13. Houghton RL, Benson DR, Reynolds LD, McNeill PD, Sleath PR, Lodes MJ, et al. A multi-epitope synthetic peptide and recombinant protein for the detection of antibodies to Trypanosoma cruzi in radioimmunoprecipitationconfirmed and consensus-positive sera. J Infect Dis. 1999;179(5):1226-34.

14. Mucci J, Carmona SJ, Volcovich R, Altcheh J, Bracamonte E, Marco JD, et al. Next-generation ELISA diagnostic assay for Chagas disease based on the combination of short peptidic epitopes. PLoS Negl Trop Dis. 2017;11(10): e0005972.

15. Santos FL, Celedon PA, Zanchin NI, Souza WV, Silva ED, Foti L, et al. Accuracy of chimeric proteins in the serological diagnosis of chronic Chagas disease - a phase II study. PLoS Negl Trop Dis. 2017;11(3):e0005433.

16. Santos FL, Campos AC, Amorim LD, Silva ED, Zanchin NI, Celedon PA, et al. Highly accurate chimeric proteins for the serological diagnosis of chronic Chagas disease: a latent class analysis. Am J Trop Med Hyg. 2018;99(5):1174-9.

17. Santos FL, Celedon PA, Zanchin NI, Leitolis A, Crestani S, Foti L, et al. Performance assessment of a Trypanosoma cruzi chimeric antigen in multiplex liquid microarray assays. J Clin Microbiol. 2017;55(10):2934-45.

18. Santos FL, Celedon PA, Zanchin NI, Brasil TA, Foti L, Souza WV, et al. Performance assessment of four chimeric Trypanosoma cruzi antigens based on antigen-antibody detection for diagnosis of chronic Chagas disease. PLoS One. 2016;11(8):e0161100.

19. Brito VS, Santos FL, Gonçalves NL, Araujo TH, Nascimento DS, Pereira FM, et al. Performance of commercially available serological screening tests for human T-cell lymphotropic virus infection in Brazil. J Clin Microbiol. 2018; 56(12):e00961-18.

20. Leeflang MM, Allerberger F. How to: evaluate a diagnostic test. Clin Microbiol Infect. 2019;25(1):54-9.

21. Cohen JF, Korevaar DA, Altman DG, Bruns DE, Gatsonis CA, Hooft L, et al. STARD 2015 guidelines for reporting diagnostic accuracy studies: explanation and elaboration. BMJ Open. 2016:6(11):e012799.

22. Tibayrenc M, Ayala FJ. Towards a population genetics of microorganisms: the clonal theory of parasitic protozoa. Parasitol Today. 1991;7(9):228-32.

23. Zingales B, Andrade SG, Briones MR, Campbell DA, Chiari E, Fernandes O, et al. A new consensus for Trypanosoma cruzi intraspecific nomenclature: second revision meeting recommends Tcl to TcVI. Mem Inst Oswaldo Cruz. 2009;104(7):1051-4.

24. Zingales B, Miles MA, Campbell DA, Tibayrenc M, Macedo AM, Teixeira MM, et al. The revised Trypanosoma cruzi subspecific nomenclature: rationale, epidemiological relevance and research applications. Infect Genet Evol. 2012;12(2):240-53.

25. Umezawa ES, Bastos SF, Camargo ME, Yamauchi LM, Santos MR, Gonzalez A et al. Evaluation of recombinant antigens for serodiagnosis of Chagas' disease in south and Central America. J Clin Microbiol. 1999:37(5):1554-60.

26. Umezawa ES, Bastos SF, Coura JR, Levin MJ, Gonzalez A, Rangel-Aldao R, et al. An improved serodiagnostic test for Chagas' disease employing a mixture of Trypanosoma cruzi recombinant antigens. Transfusion. 2003;43(1): $91-7$.

27. Verani JR, Seitz A, Gilman RH, LaFuente C, Galdos-Cardenas G, Kawai V, et al. Geographic variation in the sensitivity of recombinant antigen-based rapid tests for chronic Trypanosoma cruzi infection. Am J Trop Med Hyg. 2009; 80(3):410-5

28. Martin DL, Marks M, Galdos-Cardenas G, Gilman RH, Goodhew B, Ferrufino L, et al. Regional variation in the correlation of antibody and T-cell responses to Trypanosoma cruzi. Am J Trop Med Hyg. 2014;90(6):1074-81.

29. Abras A, Gállego M, Muñoz C, Juiz NA, Ramírez JC, Cura Cl, et al. Identification of Trypanosoma cruzi discrete typing units (DTUs) in LatinAmerican migrants in Barcelona (Spain). Parasitol Int. 2017;66(2):83-8.

Ready to submit your research? Choose BMC and benefit from:

- fast, convenient online submission

- thorough peer review by experienced researchers in your field

- rapid publication on acceptance

- support for research data, including large and complex data types

- gold Open Access which fosters wider collaboration and increased citations

- maximum visibility for your research: over $100 \mathrm{M}$ website views per year

At BMC, research is always in progress.

Learn more biomedcentral.com/submissions 